\title{
Voltage and Congestion Control in Active Distribution Networks Using Fast Sensitivity Analysis
}

\author{
Khaled Alzaareer \\ École de technologie supérieure \\ Montreal, Quebec, Canada
}

Claude Ziad EL-BAYEH
Concordia University

\author{
Maarouf Saad \\ École de technologie supérieure \\ Montreal, Quebec, Canada
}

Dalal Asber

Research Institute of Hydro-Quebec Montreal, Quebec, Canada

\author{
Hasan Mehrjerdi \\ Qatar University \\ Doha, Qatar
}

\begin{abstract}
Active distribution networks will meet new challenges in voltage and congestion control due to the high penetration levels of distributed generations (DG). Optimal voltage control techniques are mainly based on the relation between the network voltages and control variables (i.e. power injection). Thus, in this paper, a centralized real time scheme using a fast method for computing the sensitivity coefficients is presented to control congestion and voltage problems. The sensitivity coefficients of network voltages as well as the branch line currents are obtained via direct analytical derivation. A $11 \mathrm{KV}, 75-b u s$ distribution system including several DGs is used to validate the proposed method. Simulation results show that the proposed method is able to maintain the network voltages and line currents within acceptable limits.
\end{abstract}

Keywords - Sensitivity analysis, distribution networks, voltage control, thermal limits, distributed generation.

\section{INTRODUCTION}

The increase in the penetration levels of renewable distributed generation connected to distribution networks will cause a rise in network operational problems. The violations in network voltages and branch thermal limits are the main problems associated with integration of DG units. The development of Smart Grid technology makes it easier to propose a centralized scheme to control the voltage and congestion problems. Voltage control methods are mainly divided into centralized and decentralized schemes [1]. The method proposed in [2] and [3] is one of the previous works that deals with voltage problems in active distribution networks through a centralized scheme. The method depends on the model predictive control to obtain a global voltage control. The methods in [4] and [5] deals with thermal problems. The method is based on the "last-in first-off" principle to control the congestion problems. The authors in [6] proposed a control scheme for both voltage and thermal problems. The approach is based on a sensitivity-based model of the network. One of the disadvantages of centralized scheme-based voltage control methods is that they require high computing time. The sensitivity analysis method that is used to find the sensitivity coefficients of network voltages and branch currents with respect to control variables plays a main role in the computation time. Voltage control approaches, even the recent ones, are mainly based on the traditional methods for voltage sensitivity analysis [2], [7]. [8], [9]. The well-known sensitivity method is by using the inverse of Jacobian matrix [10]. This sensitivity method depends on the load flow study. However, the extracted sensitivities are required to be updated as the network operating condition is changed. This increases the computational time and, therefore, add a new challenge for online voltage and congestion control in distribution networks in the presence of DG units. Many other sensitivity approaches are developed in the literature [11],[12],[13],[14]. However, these methods suffer from inaccuracy and/or low computational speed.

In this work, an online voltage control method for active distribution networks and based on a fast sensitivity analysis is presented. The target of this work is to control both the network voltages and congestion problems. The proposed method keeps the branch currents smaller than their maximum capacity when output power of DG units varies. For online requirements, especially in the context of optimization algorithms, the voltages and branch currents are evaluated using an efficient computation for sensitivity analysis. This paper is organized as follows. Section II presents the sensitivity analysis to find the sensitivity coefficients of network voltages as well as the branch line currents. Section III formulates the optimization problem for the voltage control considering the thermal limits. Simulation results are presented in section IV while Section V states the conclusions.

\section{The Proposed SENSITIVITY ANALYSIS}

Fig. 1 represents a two-bus system where the load at bus 2 is $\mathrm{P}+\mathrm{jQ}$ and the node voltage and current are $\mathrm{V}$ and $\mathrm{I}$ respectively.

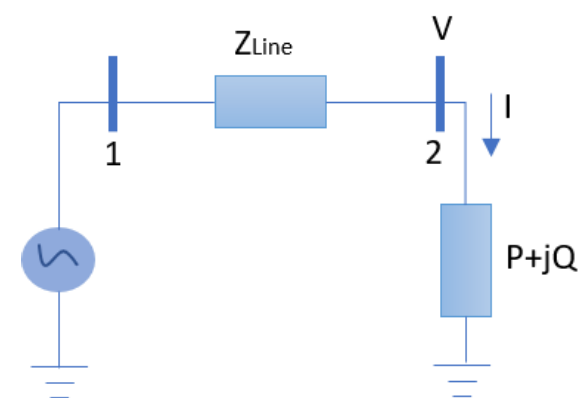

Fig.1. Two-bus system

The load at bus $2(\mathrm{~S})$ can be calculated as:

$$
\mathrm{S}=V I^{*}
$$

The node current I can be found in terms of the network voltages according to (2). 


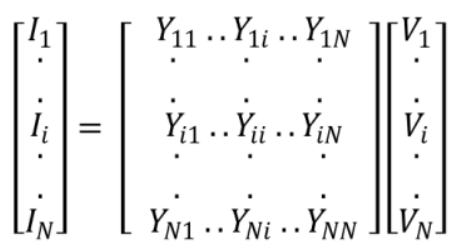

Where $\mathrm{Y}$ represents the network bus admittance, I and $\mathrm{V}$ represent bus current and voltage vectors, respectively. $\mathrm{N}$ represents the number of network buses. Accordingly, the current $\mathrm{I}^{*}$ of bus $\mathrm{i}$ can be expressed as

$$
I_{i}^{*}=\sum_{j \in N} Y_{i j}^{*} V_{j}^{*}
$$

By substituting (3) into (1) and taking the derivative of (1) with respect to the control variable $\mathrm{u}$, we obtain :

$$
\frac{\partial S}{\partial u}=V \sum_{\mathrm{j} \epsilon M} Y_{i j}{ }^{*} \frac{\partial V_{j}^{*}}{\partial u}+\frac{\partial V}{\partial u} \sum_{\mathrm{j} \in N} Y_{i j}{ }^{*} V_{j}^{*}
$$

Where $\mathrm{M}$ is the number of PQ buses.

The expression in (4) can be extended for multi-bus systems as:

$$
\frac{\partial S_{i}}{\partial u_{x}}=V_{i} \sum_{\mathbf{j} \in M} Y_{i j}{ }^{*} \frac{\partial V_{j}^{*}}{\partial u_{x}}+\frac{\partial V_{i}}{\partial u_{x}} \sum_{\mathbf{j} \in N} Y_{i j}{ }^{*} V_{j}^{*}
$$

Where $\mathrm{i}$ denotes for the $\mathrm{i}^{\text {th }}$ PQ bus while $u_{x}$ represents the $\mathrm{x}^{\text {th }}$ control variable. represents the partial derivative of the complex power of bus $i$ with respect to the $x^{\text {th }}$ control variable. represents the partial derivative of the voltage of bus $i$ with respect to the $\mathrm{x}^{\text {th }}$ control variable. $V_{i}$ represent the node voltage of bus $i$ and $*$ denotes for the conjugate. In this paper, the nodal injections of active and reactive power (i.e. the power produced by DG units) are considered as the main voltage controls for active distribution networks. Thus, the partial derivates in (5) can be expressed with respect to real or reactive power injected at the $x^{\text {th }}$ bus (i.e.). The sensitivity can be found as:

$$
\frac{\partial S_{i}}{\partial P_{x}}=\left\{\begin{array}{cc}
1 & i=x \\
0 & \text { otherwise }
\end{array}\right.
$$

The sensitivity $\frac{\partial S_{i}}{\partial Q_{x}}$ can be found as:

$$
\frac{\partial S_{i}}{\partial Q_{x}}=\left\{\begin{array}{cr}
-j 1 & i=x \\
0 & \text { otherwise }
\end{array}\right.
$$

By performing (5) for each and solving the linear system, we can obtain the partial derivatives of voltage (or voltage conjugates). The voltage sensitivities can be obtained according to the relation expressed in (8) as:

$$
\frac{d\left|V_{i}\right|}{d u_{x}}=\frac{1}{\left|V_{i}\right|}\left(\operatorname{Re}\left(V_{i}\right) \frac{d\left(\operatorname{Re}\left(V_{i}\right)\right)}{d u_{x}}+\operatorname{Im}\left(V_{i}\right) \frac{d\left(\operatorname{Im}\left(V_{i}\right)\right)}{d u_{x}}\right)
$$

To consider the thermal limits in active distribution network, it is necessary to find the sensitivities of branch currents to the control variable $u_{x}$. Since the branch current can be expressed as we obtain that:

$$
\frac{d I_{i j}}{d u_{x}}=Y_{i j}\left(\frac{d V_{i}}{d u_{x}}-\frac{d V_{j}}{d u_{x}}\right)
$$

The branch current coefficients can be obtained as:

$$
\frac{d\left|I_{i j}\right|}{d u_{x}}=\frac{1}{\left|I_{i j}\right|}\left(\operatorname{Re}\left(I_{i j}\right) \frac{d\left(\operatorname{Re}\left(I_{i j}\right)\right)}{d u_{x}}+\operatorname{Im}\left(I_{i j}\right) \frac{d\left(\operatorname{Im}\left(I_{i j}\right)\right)}{d u_{x}}\right)
$$

\section{VOLTAGE CONTROL SCHEME}

In this study, the optimal control problem is formulated by taking the advantage of computing the voltage and branch currents sensitivity coefficients via a fast method. The controlled variables are the network voltages and branch currents while the controls are the active and reactive power produced by DG units. The voltage control approach proposed in this study is based on a multi-step optimization proposed in [2] to smoothly correct the voltages over interval of n discrete steps. This control scheme can be implemented in a control center for a global decision. Since this work aims to minimize the change in the power produced by DG units, the following optimization problem can be used:

$$
\begin{gathered}
O b j 1=\min \sum_{k=0}^{n-1}\|\Delta Q(t+k)\|_{F}^{2}+\|\varepsilon\|_{H}^{2} \\
O b j 2=\min \sum_{k=0}^{n-1}\|\Delta P(t+k)\|_{G}^{2}+\|\Delta Q(t+k)\|_{F}^{2} \\
+\|\varepsilon\|_{H}^{2}
\end{gathered}
$$

Subject to:

$$
\begin{aligned}
& -\varepsilon_{1} A+\left|V_{i}\right|^{\min } \leq\left|V_{i}\right|(t+k) \leq\left|V_{i}\right|^{\max }+\varepsilon_{2} A \\
& \left|I_{i j}\right|(t+k) \leq\left|I_{i j}\right|^{\max }+\varepsilon_{3} A \\
& \Delta Q_{x}{ }^{\min } \leq \Delta Q_{x}(t+k) \leq \Delta Q_{x}{ }^{\max } \\
& \Delta P_{x}^{\min } \leq \Delta P_{x}(t+k) \leq \Delta P_{x}^{\max } \\
& P_{x}{ }^{\min } \leq P_{x}(t+k) \leq P_{x}^{\max } \\
& Q_{x}{ }^{\min } \leq Q_{x}(t+k) \leq Q_{x}^{\max }
\end{aligned}
$$

Where represents the vector of changes of real and reactive power injected by DGs respectively. represents the vector of slack variables. 'A' represents a unitary vector., $\mathrm{F}$ and $\mathrm{H}$ are weight matrices used to distinguish between the expensive and cheap variables. is the predicted voltage magnitude of bus i given the measurements at time instant $t$. is the predicted branch current. are the voltage sensitivities of bus $i$ with respect to the real and reactive power injections at the $\mathrm{x}^{\text {th }}$ bus. are the branch current sensitivities of line "ij" with respect to the real and reactive power injections at the $\mathrm{x}^{\text {th }}$ bus. represents the maximum current limit for the branch "ij". are the real and reactive power injected by the $x^{\text {th }} \mathrm{DG}$, respectively. are the change in real and reactive power injected by the $x^{\text {th }} D G$, respectively. "min" and "max" denote for the minimum and maximum limits, respectively.

In this work, the optimal voltage control is found firstly according to the objective function in (11.a). If the network voltages and branch line currents are brought back inside the normal voltage range, then the DG units have sufficient 
reactive power capability for control and thus no real power curtailment is required. If not, the active power output by DG units is needed according to objective function (11.b), to avoid the violation in the voltages.

It is worth mentioning that the active power generated by DG units are constrained by a capacity (in case of conventional DG units) or weather conditions (in case of renewable sources). Thus, the controller cannot request more than the active power that is being generated. The reactive power produced by DG units are considered cheap controls while their output active power is considered expensive controls and should not be requested unless the DG units fails to control the voltage and congestion by injection reactive power. Thus, active powers of the DG units have a high weight to minimize their use. Voltage and current sensitivities provide us the information of how much changing active or reactive power produced by DG units can affect the network voltages or line branch currents. Using the sensitivity coefficients, the DG units which have higher effects on the violated voltage are selected.

The node voltage and line currents constraints in (11) can be obtained by performing power flow calculations with the updated power changes of DG units. Thus, it is necessary to reduce the consuming time of the frequent power flow computations. For this purpose, the network voltages and line currents are evaluated based on a voltage sensitivity matrix without needing to use all the network data in the optimization process. Reducing the calculation time is very important for its real-time applications. Hence, the voltage and current sensitivity coefficients obtained in section II are used to update the network voltages and line currents as follows:

$$
\begin{gathered}
\left|V_{i}\right|(t+k)=\left|V_{i}\right|(t+k-1)+\sum_{x=0}^{m} \frac{\partial\left|V_{i}\right|}{\partial P_{x}} \Delta P_{x}(t+k) \\
+\sum_{x=0}^{m} \frac{\partial\left|V_{i}\right|}{\partial Q_{x}} \Delta Q_{x}(t+k) \\
\left|I_{i j}\right|(t+k)=\left|I_{i j}\right|(t+k-1)+\sum_{x=0}^{m} \frac{\partial\left|I_{i j}\right|}{\partial P_{x}} \Delta P_{x}(t+k) \\
+\sum_{x=0}^{m} \frac{\partial\left|I_{i j}\right|}{\partial Q_{x}} \Delta Q_{x}(t+k)
\end{gathered}
$$

Where $\mathrm{m}$ is number of DG units is the measured voltage magnitude of bus $i$. is the measured current magnitude of branch "ij".

\section{TEST System AND Simulation RESUlts}

In this paper, a 75 -bus, $11 \mathrm{kV}$ distribution system shown in Fig. 2 is used to examine the proposed method. The network consists of 22DG units and 53 load bus. The network parameters and data can be found in [15]. This network and the voltage control method have been simulated in
MATLAB/SIMULINK. MATLAB was also interfaced to LINGO software to solve the optimization problem.

In this work, it is assumed that the maximum voltage limit is $1.04 \mathrm{p}$.u while the minimum voltage limit is $0.98 \mathrm{p}$.u. It is assumed that the rating for DG units are 4MVA. The weights for active power changes are set to be 40 times the weights for reactive power changes. The weights for the slack variables is 900 times the weight for reactive power changes.

The line branches in which the thermal limits are considered in this paper are the branches that connect DG units with the network. Indeed, the current variations can be caused by reactive power changes of DG units. To take into account the branch thermal limits, it is assumed that the branch currents should be less than its acceptable maximum value.

\section{A. Case 1: undervoltages}

In the first case, the load demands are assumed to be $100 \%$ of their nominal values while DG units are operated at $10 \%$ of their rated powers. This cause undervoltage at some network nodes. In this case, the reactive powers of DG units are enough to correct the voltage and congestion problems and, thus, active power curtailment is not used. The sensitivities of the network voltages and line branch currents with respect to the real and reactive power output by DG units are computed by (12) and (13) respectively.

Fig. 3 shows the network load voltages without any control and the controlled voltages by using the proposed control method. The figure shows that the undervoltages have been efficiently eliminated by using the voltage control method via a fast sensitivity analysis method.

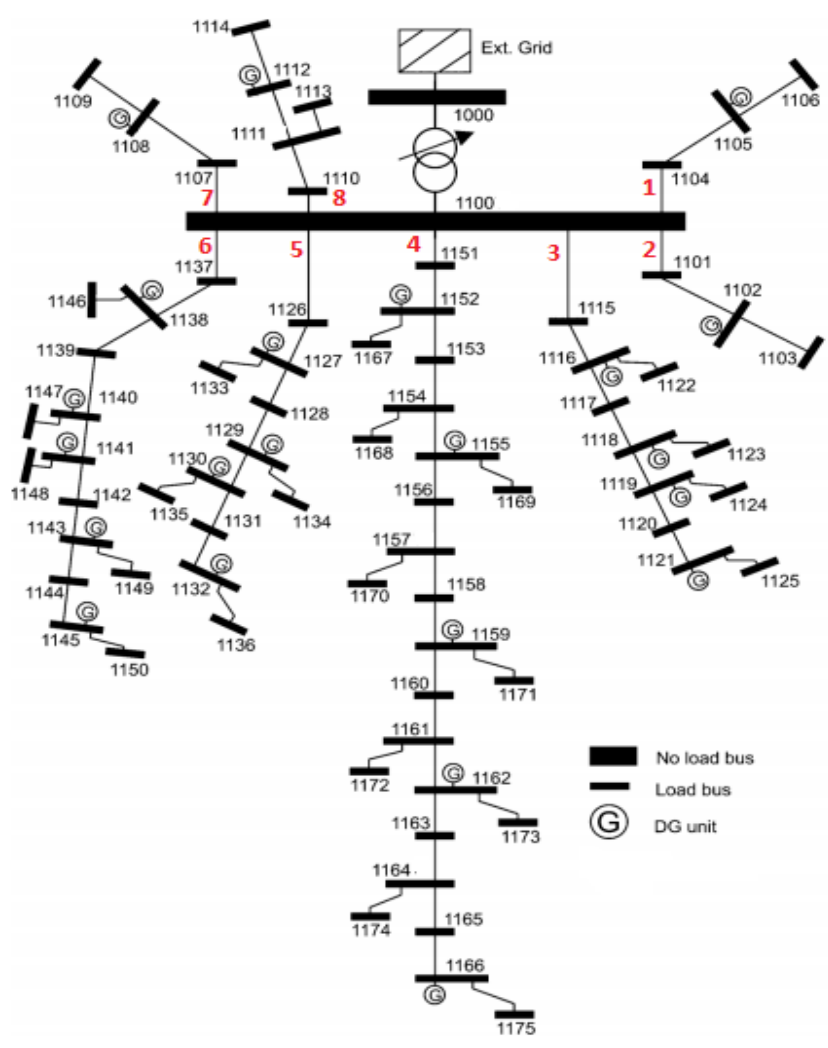

Fig.2. Topology of the test network [2] 


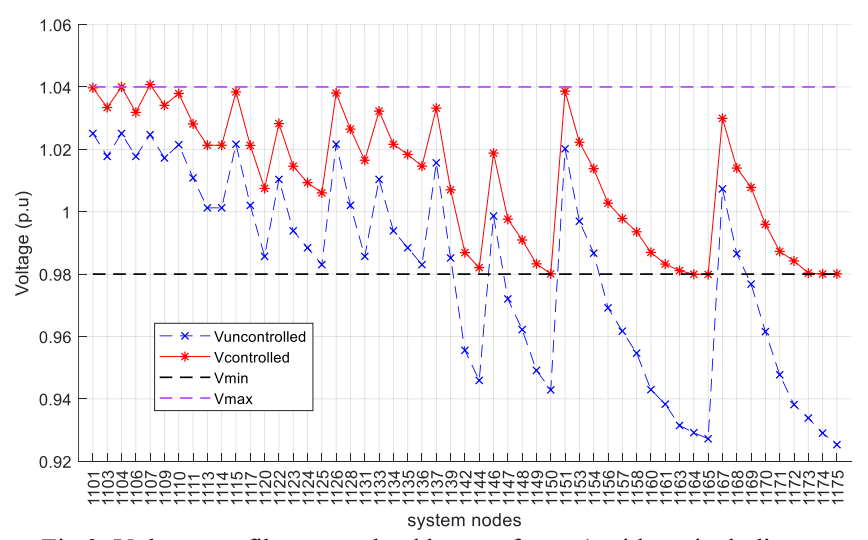

Fig.3. Voltage profile among load buses of case 1 without including thermal limits.

Figure 4 shows the reactive power changes of DG units in order to solve the voltage and congestion problems. In general, the voltage control method tries to utilize the most efficient DG unit for voltage and congestion control with its maximum possible capacity. If this is not enough, then, the next most efficient units will be used. In this case, the most violated voltage occurs at bus 1175 . The nearest DG unit, located at bus 1166 , has the higher effect on the voltage at bus 1175. Thus, we can see from Fig. 4 that bus 1166 provides a higher amount of reactive power than other DG units.

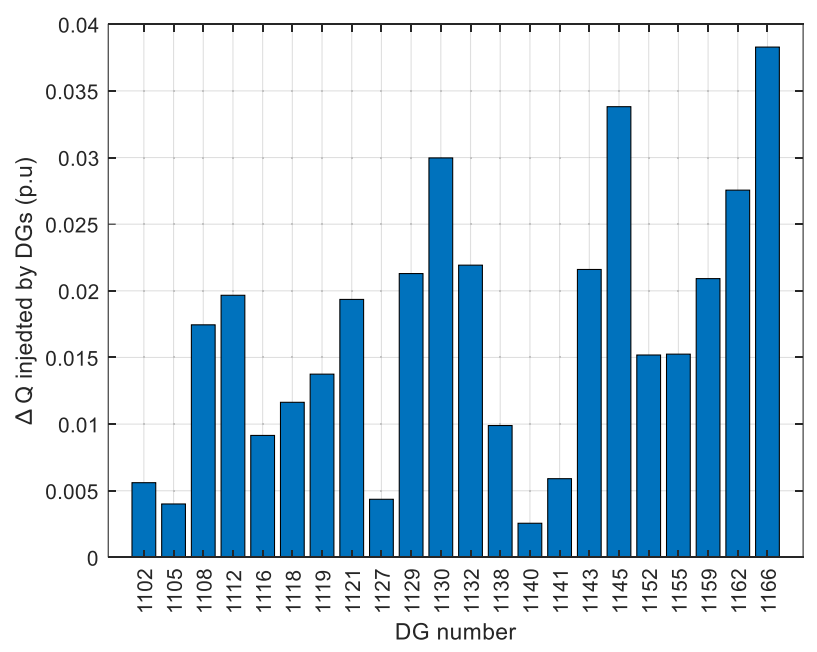

Fig.4. The change in the reactive power output by DG units of case 1 without including thermal limits.

It is worth mentioning that the branch thermal limits were not violated and, thus, the same results will be obtained when considering the thermal limits.

\section{B. Case 2: Overvoltages}

In this scenario, the load demands are assumed to be $10 \%$ of their nominal values while DG units are operated at $90 \%$ of their rated powers. A step increase of $5 \%$ of the Thevenin equivalent voltage (i.e. the system is affected by a disturbance in the high voltage side) is also done to make successive overvoltage. This also causes a violation in the thermal limits of some branch currents. In this scenario, reactive powers of DG units are also enough to correct the voltages without needing for changing the active power produced by DG units. The sensitivities coefficients are also computed using (12) and (13).
Fig. 5 shows the system voltages without any control and the controlled node voltages by using the proposed voltage control method. The figure also shows the comparison between the voltages when considering/not considering the thermal limits of the line branches. In this scenario, the power absorbed by DG units causes a noticeable increase in the branch currents so thermal limits of some lines are violated.

It can be seen from Fig. 5 that the overvoltages at some nodes have been efficiently eliminated. The figure also shows that there is a difference in the controlled voltage when considering / not considering the thermal limits. This is due to the difference in the reactive power outputs by DG units during the two cases: considering and ignoring thermal limits.

Figure 6 shows the reactive power changes of DG units in order to solve the voltage and congestion problems. The comparison between the changes in reactive powers when considering/ ignoring the thermal limits are also illustrated. By ignoring the branch thermal limits, some DG units can generate the requested power up to its maximum power capacity.

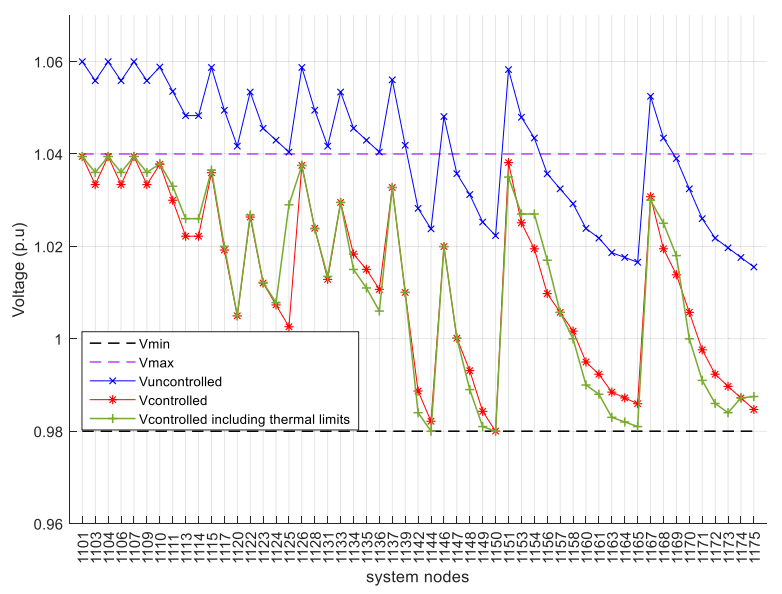

Fig.5. Voltage profile among load buses of case 2 (with/without including thermal limits).

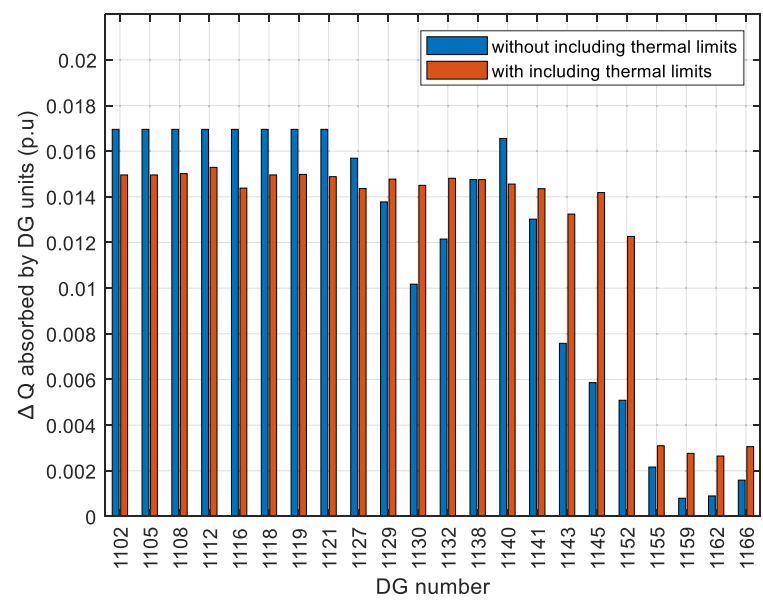

Fig.6. The change in the reactive power output by DG units of case 1 (with/without including thermal limits).

In this case, the voltage control method does not freely employ DG units at the bus with the highest voltage sensitivity index. The DG units are operated up to the branch 
thermal limits. Then, the needed remaining amount of the reactive power is supplied by other DG units. From Fig.5, we can see that when the thermal limits are included in the problem, a higher total amount of power is required to correct the same violated voltages. This is due to fact that power injection at a particular node has a higher impact on its voltage than power injections at other buses.

According to the simulation results, we can conclude that the sensitivity coefficients were successfully validated through the optimization-based voltage control.

It is worth mentioning that this work uses multi-step optimization since it is hard to obtain a feasible solution for large networks by only one step optimization. However, the dynamic simulation of multi-step optimization can be found in [2].

\section{CONCLUSIONS}

In this work, a centralized method for voltage and congestion control of active medium-voltage distribution networks are presented. The method depends on the sensitivity analysis to evaluate the network voltages as well as the branch currents inside the optimization process. The sensitivity analysis approach is fast and does not require to update the sensitivity coefficients through power flow as in Jacobian based method.

The results showed that the voltage control method is able to determine the optimal changes of the power produced by DG units in order to correct the network voltages while maintaining the thermal limits of the network branches less than their maximum limits. The proposed method is fast and can be used in online applications of the system. It does not require complex calculations and can effectively improve the calculation efficiency. The voltage control method can also effectively meet the need of the continuous variation of the load and/or generation of the system.

The voltage control method can be extended in the next works to include all control variables (as On-load tap changers) in the control process. The performance of the voltage control approach in the case of changing network parameters and topology under different congestion types will also be studied. The dynamic simulation for congestion results is also one of our future targets.

\section{ACKNOWLEDGMENT}

This work was supported by the National Priorities Research Program under Grant 11S-1125-170027 from the Qatar National Research Fund (a member of Qatar Foundation).

\section{REFERENCES}

[1] V. A. Evangelopoulos, P. S. Georgilakis, and N. D. Hatziargyriou, Optimal operation of smart distribution networks: A review of models, methods and future research, Electric Power Systems Research, 140 pp. 95-106, 2016.

[2] G. Valverde, T. Van Cutsem, "Model Predictive Control of Voltages in Active Distribution Networks" IEEE Trans. on Smart Grid, vol.4, no.4, pp.2152-2161, 2013.

[3] T. Van Cutsem and G. Valverde, "Coordinated Voltage Control of Distribution Networks Hosting Dispersed Generation”, Proc. 22nd International Conference on Electricity Distribution (CIRED), Stockholm, 2013.

[4] M. J. Dolan, E. M. Davidson, I. Kockar, G. W. Ault, and D. J. McArthur, "Distribution Power Flow Management Utilizing an Online Optimal Power Flow Technique," IEEE Trans. On Power Syst., vol. 27, no. 2, pp. 790-799, 2012.

[5] R. A. F. Currie, G. W. Ault, R. W. Fordyce, D. F. MacLeman, M. Smith, and J. R. McDonald, "Actively managing wind farm power output," IEEE Trans. on Power Syst., vol. 23, no. 3, pp. 1523-1524, 2008.

[6] T. Sansawatt, L. F. Ochoa, G. P. Harrison, "Smart Decentralized Control of DG for Voltage and Thermal Constraints Management," IEEE Trans. on Power Syst., vol. 27, no. 3, pp. 1637-1645, 2012,

[7] Q. Zhou, and J. W. Bialek, "Generation curtailment to manage voltage constraints in distribution networks," IET Generation Transmission Distribution, vol. 1, no. 3, 2007.

[8] A. Borghetti, M. Bosetti, S. Grillo, M. Paolone, and F. Silvestro, "Short-term scheduling of active distribution systems," 2009 IEEE Power Tech Conference, Romania, 2009.

[9] Zhao, B., Xu, Z., Xu, C., Wang, C., \& Lin, F. "Network partition-based zonal voltage control for distribution networks with distributed PV systems, " IEEE Transactions on Smart Grid, 9(5), pp. 4087-4098, 2017.

[10] M. BegovicandA. Phadke, "Control of voltage stability using sensitivity analysis," Power Systems, IEEE Transactions on, vol. 7, no. 1, pp. 114-123, 1992

[11] Q. Zhou and J. Bialek, "Simplified calculation of voltage and loss sensitivity factors in distribution networks," in Proc. of the 16th Power Systems Computation Conference, Glasgow, Scotland, 2008.

[12] S. Conti, S. Raiti, and G. Vagliasindi, "Voltage sensitivity analysis in radial mv distribution networks using constant current models," in Industrial Electronics (ISIE), 2010 IEEE International Symposium on. IEEE, pp. 2548-2554, 2010

[13] D. Khatod, V. Pant, and J. Sharma, "A novel approach for sensitivity calculations in the radial distribution system," Power Delivery, IEEE Transactions on, vol. 21, no. 4, pp. 2048-2057, 2006.

[14] R. Gurram and B. Subramanyam, "Sensitivity analysis of radial distribution network-adjoint network method," International Journal of Electrical Power \& Energy Systems, vol. 21, no. 5, pp. 323-326, 1999.

[15] United Kingdom Generic Distribution Network (UKGDS). 